\title{
Energy relaxation in nonlinear one-dimensional lattices
}

\author{
R. Reigada \\ Departament de Química Física, Universitat de Barcelona, Avenida Diagonal 647, 08028 Barcelona, Spain
}

A. Sarmiento

Instituto de Matemáticas, Universidad Nacional Autónoma de México, Avenida Universidad s/n, 62200 Chamilpa, Morelos, Mexico

\author{
Katja Lindenberg \\ Department of Chemistry and Biochemistry and Institute for Nonlinear Science, University of California, \\ San Diego, La Jolla, California 92093
}

(Received 8 August 2001; published 19 November 2001)

\begin{abstract}
We study energy relaxation in thermalized one-dimensional nonlinear arrays of the Fermi-Pasta-Ulam type. The ends of the thermalized systems are placed in contact with a zero-temperature reservoir via damping forces. Harmonic arrays relax by sequential phonon decay into the cold reservoir, the lower-frequency modes relaxing first. The relaxation pathway for purely anharmonic arrays involves the degradation of higher-energy nonlinear modes into lower-energy ones. The lowest-energy modes are absorbed by the cold reservoir, but a small amount of energy is persistently left behind in the array in the form of almost stationary low-frequency localized modes. Arrays with interactions that contain both a harmonic and an anharmonic contribution exhibit behavior that involves the interplay of phonon modes and breather modes. At long times relaxation is extremely slow due to the spontaneous appearance and persistence of energetic high-frequency stationary breathers. Breather behavior is further ascertained by explicitly injecting a localized excitation into the thermalized arrays and observing the relaxation behavior.
\end{abstract}

DOI: 10.1103/PhysRevE.64.066608

PACS number(s): 05.45.-a, 05.40.-a, 63.20.Pw

\section{INTRODUCTION}

The localization of vibrational energy in discrete nonlinear arrays has attracted a huge amount of interest in the past 15 years as a possible mechanism for the efficient storage and transport of energy. The reasons for the broad interest in these phenomena are at least twofold: on the one hand, they embody many of the interesting effects of the interplay of nonlinearity, discretization, and stochasticity, and on the other they may be of use in explaining a variety of physical and biophysical phenomena. The interest in nonlinear arrays, serving as energy storage and transfer assemblies for chemical or photochemical processes, is not uncommon [1], and literature on the subject goes back for two decades [2]. More recently, the localization and transport of vibrational energy has been invoked in a number of physical settings including DNA molecules [3], hydrocarbon structures [4], the creation of vibrational intrinsic localized modes in anharmonic crystals [5], photonic crystal waveguides [6], and targeted energy transfer between donors and acceptors in biomolecules [7].

Many types of nonlinear arrays exhibit spontaneous localization, and in each of these the conditions that lead to localization are complex and multifaceted. A vast literature deals not only with different types of arrays but with issues such as boundary conditions, initial conditions, whether the system is closed or forced, thermal effects, etc. It is impossible to present here a full catalog or even sensibly organized panorama of results; a number of excellent reviews have aided greatly in the effort $[8,9]$.

Increasing attention has recently been devoted to thermalized nonlinear arrays. Whether energy can be transmitted without the destructive thermal and dispersive degradation that occurs in harmonic lattices is clearly an important question: one thinks, for example, about the amazing energytransfer cascade occurring in the photosynthetic process [10], or about the ability of some proteins to efficiently store and transport and thereby convert chemical energy into mechanical energy [11-13]. Transport across a thermal gradient and questions concerning the validity of the usual Fourier law have very recently been addressed in a number of papers [14-17]. Relaxation to thermal equilibrium and the nature of this equilibrium have been addressed in recent work [1821], as has the transport of energy in thermal arrays [22-26].

In addition to the phonons associated with the linear portion of the potential in a nonlinear array, a variety of stationary, and nonstationary but long-lived excitations are possible, including solitons $[17,24,25,27-29]$ (long-wavelength excitations that persist from the continuum limit upon discretization), periodic breathers $[8,9,21,24,25,28-37]$ (spatially localized time periodic excitations that persist from the anticontinuous limit upon coupling), and so-called chaotic breathers [21] (localized excitations that evolve chaotically). These nonlinear excitations arise (even spontaneously) and survive for a long time in numerical experiments, and they clearly play an important role in determining the global macroscopic properties of nonlinear extended systems.

The nonlinearity in discrete nonlinear arrays may occur in the interactions $V\left(x_{i}, x_{j}\right)$ and/or in the "local" or "external" potentials $U\left(x_{i}\right)$. Here $x_{i}$ is the displacement of particle $i$ from its equilibrium position. While the presence of local potentials favors energy localization, we are interested in localized energy that can also move, and mobility tends to be easier in the absence of a local potential $[20,22,23]$. We therefore focus on Fermi-Pasta-Ulam (FPU) lattices of unit 
masses, each connected to its nearest neighbors by quadratic and/or quartic springs (FPU $\beta$ model). Here we deal only with the one-dimensional problem (the two-dimensional FPU system will be considered elsewhere [38]). The Hamiltonian of the system is

$$
H=\sum_{i=1}^{N} \frac{\dot{x}_{i}^{2}}{2}+\frac{k}{2} \sum_{i=1}^{N}\left(x_{i}-x_{i-1}\right)^{2}+\frac{k^{\prime}}{4} \sum_{i=1}^{N}\left(x_{i}-x_{i-1}\right)^{4},
$$

where $N$ is the number of sites. $k$ and $k^{\prime}$ are the harmonic and anharmonic force constants, respectively. In this work we focus on the thermal relaxation of FPU chains. Thermal relaxation and associated mobility properties turn out to be entirely different in purely harmonic $\left(k^{\prime}=0\right)$, purely anharmonic $(k=0)$, and "mixed" ( $k$ and $\left.k^{\prime} \neq 0\right)$ one-dimensional (1D) systems.

To study energy relaxation we initially thermalize the system at temperature $T$ (see below). We then connect the end sites of the system to a zero-temperature reservoir via appropriate damping terms and observe the thermal relaxation of the array toward zero temperature $[19,39,40]$. In order to understand the role of the various interactions (quadratic, quartic) and of the localized modes that spontaneously emerge in the thermalization and relaxation process, we perform a second numerical experiment where we inject, at the center of the thermalized chain, a localized breatherlike excitation of energy much higher than the thermal energy. The dynamics of such excitations have been studied in some detail in a variety of contexts, but not in thermalized arrays. Again, we observe how the thermal energy as well as the excitation energy relax toward equilibrium.

Section II describes the preparation of our system. In Sec. III we discuss the relaxation behavior of an initially thermalized chain connected to a zero-temperature reservoir. In Sec. IV we consider the relaxation behavior when a high-energy localized excitation is introduced in the thermalized chain. Section V contains a summary of the results.

\section{INITIAL CONDITIONS}

Different energy distributions in nonlinear arrays evolve quite differently $[18,21,28,41]$, and therefore existing work is not sufficient to predict the relaxation behavior of initially thermalized arrays. To thermalize the system to a given temperature $T$ we augment the equations of motion resulting from Eq. (1) with the Langevin prescription connecting each site to a heat bath:

$$
\ddot{x}_{i}=-\frac{\partial}{\partial x_{i}}\left[V\left(x_{i}-x_{i-1}\right)+V\left(x_{i+1}-x_{i}\right)\right]-\gamma_{0} \dot{x}_{i}+\eta_{i}(t) .
$$

Here $V\left(x_{i}-x_{j}\right)$ is the FPU potential, $\gamma_{0}$ is the dissipation parameter, and the $\eta_{i}(t)$ are mutually uncorrelated zerocentered Gaussian $\delta$-correlated fluctuations that satisfy the fluctuation-dissipation relation at temperature $T$ :

$$
\left\langle\eta_{i}(t)\right\rangle=0, \quad\left\langle\eta_{i}(t) \eta_{j}\left(t^{\prime}\right)\right\rangle=2 \gamma_{0} k_{B} T \delta_{i j} \delta\left(t-t^{\prime}\right) .
$$

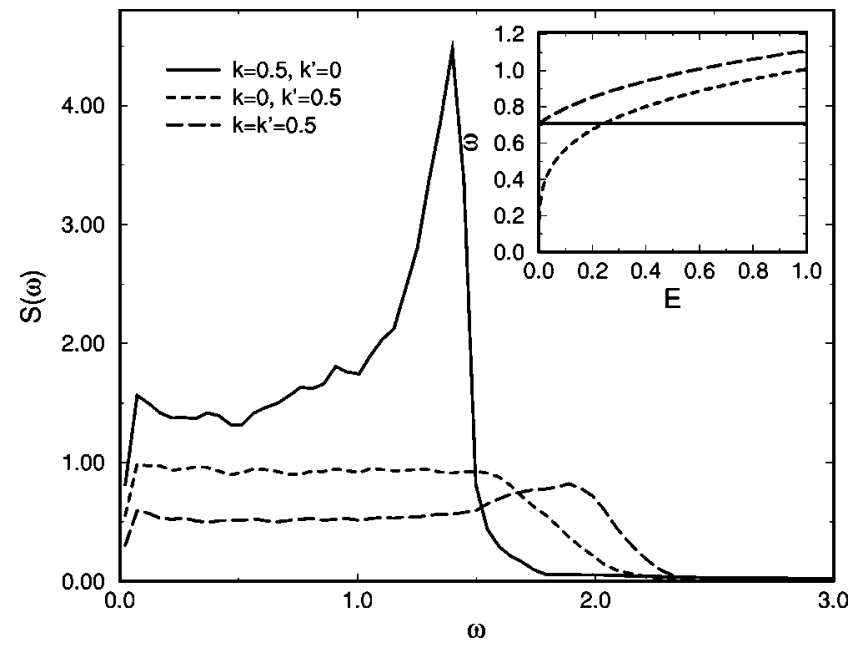

FIG. 1. Equilibrium spectrum for each of the three arrays with $N=50$ at $T=0.5$. Inset: frequency vs energy for the three potentials.

The brackets here and below denote an ensemble average, and $k_{B}$ is Boltzmann's constant. We implement free-end boundary conditions, $x_{0}=x_{1}$ and $x_{N}=x_{N+1}$, a common set of boundary conditions in relaxation studies (we do stress, however, that although boundary conditions do not strongly affect equilibrium properties, they do strongly affect some relaxation dynamics). For the integrations here and subsequently we use the fourth-order Runge-Kutta method.

The equilibrium energy landscape of our arrays can be characterized via appropriate correlation functions and/or associated frequency spectra. A convenient choice is the relative displacement autocorrelation function

$$
C(\tau)=\frac{1}{(N-1)} \sum_{i=2}^{N}\left\langle\Delta_{i}(t+\tau) \Delta_{i}(t)\right\rangle
$$

where $\Delta_{i}(t)$ is the relative displacement

$$
\Delta_{i}(t) \equiv x_{i}(t)-x_{i-1}(t)
$$

The associated spectrum is

$$
S(\omega)=2 \int_{0}^{\infty} d \tau C(\tau) \cos \omega \tau
$$

Typical equilibrium spectra for the three chains are shown in Fig. 1. The temperature and other parameters are indicated in the figure and caption. The harmonic spectrum has a peak near $\omega=\sqrt{4 k}=\sqrt{2}$, and this is also roughly the temperatureindependent spectral width. This spectrum can be calculated analytically [23], and one obtains (with periodic boundary conditions, but for sufficiently long chains the boundary conditions do not affect the thermal equilibrium spectrum):

$$
S(\omega)=\frac{4 \gamma_{0} k_{B} T}{N} \sum_{q=0}^{N-1} \frac{1-\cos (2 \pi q / N)}{\left[r_{1}^{2}(q)+\omega^{2}\right]\left[r_{2}^{2}(q)+\omega^{2}\right]},
$$

where 


$$
r_{1,2}(q)=-\frac{\gamma_{0}}{2} \pm \sqrt{\left(\frac{\gamma_{0}}{2}\right)^{2}-4 k \sin ^{2}\left(\frac{\pi q}{N}\right)}
$$

The inset in Fig. 1 shows the frequency vs energy curves obtained from the usual relation for the period of an oscillation in a potential $V(y)$,

$$
\omega(E)=\frac{2 \pi}{\tau(E)}, \quad \tau(E)=4 \int_{0}^{y_{\max }} \frac{d y}{\sqrt{2[E-V(y)]}},
$$

where the amplitude $y_{\max }$ is the positive solution of the equation $V(y)=E$. Note that the frequency $\omega=\sqrt{k}$ associated with the harmonic oscillator in the inset lies in the middle of this spectrum, and that the harmonic spectrum is temperature independent except for the overall coefficient. The temperature dependence of the nonlinear array spectra is considerably more complex. The purely hard FPU chain (shortdashed lines) shows a broader spectrum, consistent with the fact that at energies around 0.5 (our temperature), oscillator frequencies associated with a purely hard potential are higher than that of a harmonic oscillator (cf. inset). For a considerably lower energy, say $E=0.1$, the typical frequency associated with a purely hard oscillator in the inset is lower than that of a harmonic oscillator; the associated spectrum at a temperature $T=0.1$ (not shown here) is narrower than that of the harmonic chain. The mixed chain (long-dashed lines) has a broader spectrum than the harmonic or purely hard arrays at any temperature, again consistent with the inset. Whereas the harmonic array only supports extended modes (phonons), some of the frequencies for the purely hard and mixed arrays are associated with nonlinear modes that include (especially at high frequencies) localized modes. Furthermore, it should be remembered that whereas each phonon mode is characterized by a single frequency, each nonlinear mode in general involves many frequencies.

\section{RELAXATION OF THERMALIZED ARRAYS}

Relaxation of thermalized arrays from an initial temperature $T$ to zero temperature has been studied in systems with nonlinear local potentials $[39,40]$. Some aspects of thermal relaxation in FPU chains have recently been investigated [19]; our results significantly clarify and expand on these recent results. In particular, we provide more detailed insight into the mechanisms that contribute to the thermal relaxation process. We also provide a more detailed analysis of the relaxation dynamics at long times.

As in previous relaxation studies, we disconnect the thermalized array from the temperature- $T$ heat bath [i.e., we remove the $\eta_{i}(t)$ and $\gamma_{0}$ terms from the equations of motion] and connect the ends of the chain ( sites 1 and $N$ ) via a damping with rate $\gamma$ to a zero-temperature reservoir. This causes the total energy of the chain to decay through these end points. In all our simulations we set $\gamma=0.1$. We present several sets of figures (all in dimensionless units) to illustrate the relaxation behavior. Typical energy relaxation curves for arrays averaged over initial thermalized configurations are

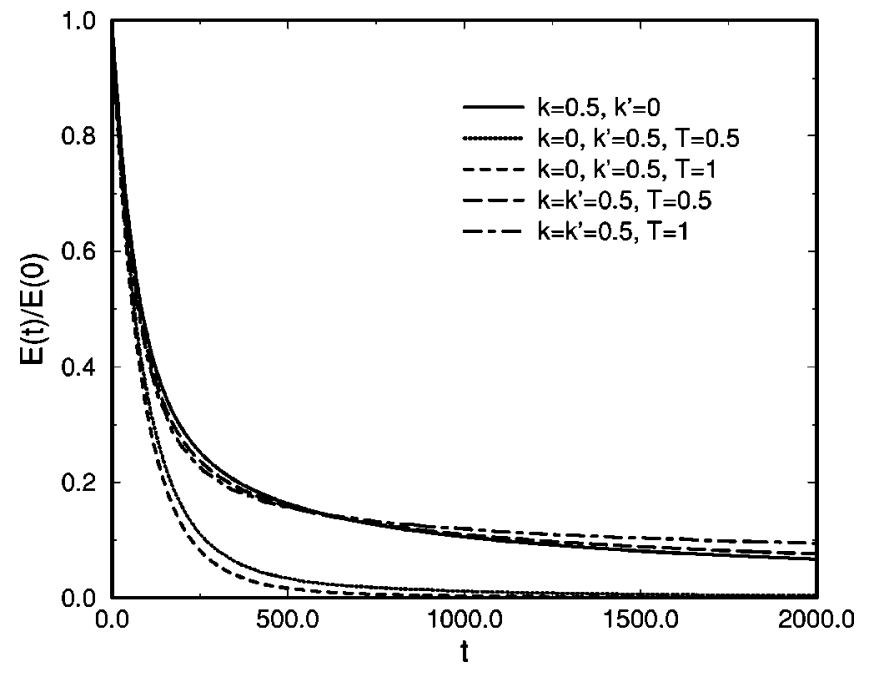

FIG. 2. Energy vs time for various relaxing arrays with $N=50$. Initially each array is in thermal equilibrium at the temperature indicated in the figure. The normalized energy of the harmonic array is independent of temperature.

shown in Fig. 2. Associated with this evolution we define the time-dependent spectra

$$
S(\omega, t) \equiv 2 \int_{0}^{\tau_{\max }} d \tau C(\tau, t) \cos \omega \tau,
$$

where $\tau_{\max } \equiv 2 \pi / \omega_{\min }$ and $\omega_{\min }$ is chosen for a desired frequency resolution; the choice $\omega_{\text {min }}=0.0982$, corresponding to $\tau_{\max }=64$, turns out to be numerically convenient. The time-dependent correlation function is actually an average over the time interval $t-t_{0}$ to $t$, where we have chosen $t_{0}$ $=100$ (short enough for the correlation function not to change appreciably but long enough for statistical purposes) and is defined as follows:

$C(\tau, t)=\frac{1}{(N-1)} \sum_{i=2}^{N} \frac{1}{\Delta t} \int_{0}^{\Delta t} d \tau^{\prime}\left\langle\Delta_{i}\left(t-\tau^{\prime}\right) \Delta_{i}\left(t-\tau^{\prime}-\tau\right)\right\rangle$,

where $\Delta t \equiv t_{0}-\tau_{\max }$. In Fig. 3 we display the evolution of the spectra for each of the chains. The spectral rendition is revealing because it clearly indicates that the decay mechanisms for harmonic and each of the anharmonic arrays are entirely different.

Several features of the energy decay curves are noteworthy. The energy in the harmonic array (solid curve in Fig. 2) is calculated by Piazza et al. [19] to be given by

$$
\frac{E(t)}{E(0)}=e^{-t / \tau_{0}} I_{0}\left(t / \tau_{0}\right)
$$

where $I_{0}$ is the modified zero-order Bessel function. At first the decay is exponential with time constant $\tau_{0}=N / 2 \gamma\left(\tau_{0}\right.$ $=250$ in the figure) and then at times larger than this time the decay changes to $\left(t / \tau_{0}\right)^{-1 / 2}$. The exponential decay time is that associated with the lowest-frequency phonon modes since they have the shortest decay times. The power-law relaxation arises from a cascade of different decay times of the 


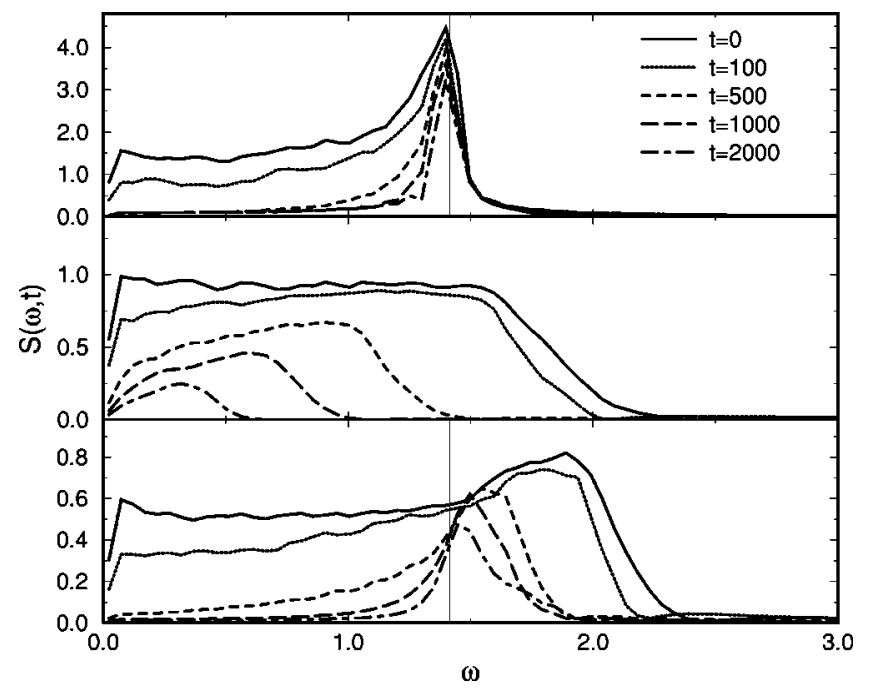

FIG. 3. Time evolution of spectra for various relaxing arrays. First panel: harmonic chain with $k=0.5$. Second panel: purely anharmonic chain with $k^{\prime}=0.5$. Third panel: mixed chain with $k$ $=k^{\prime}=0.5$. The thin vertical line indicates the frequency $\omega=\sqrt{4 k}$ $=\sqrt{2}$. Initially ( $t=0$ curves $)$ each array is in thermal equilibrium at $T=0.5$.

different phonon modes. Eventually the decay becomes exponential again when only the highest-frequency modes survive, but the energy in our chain is too low at that point to be picked up within our precision. A well-known but important point needs to be made here so that the contrasting behavior of anharmonic chains can be clarified later: the phonons in the harmonic chain are of course independent of one another, and each has to be absorbed by the cold reservoir separately. As noted above, each is absorbed on a different time scale. In

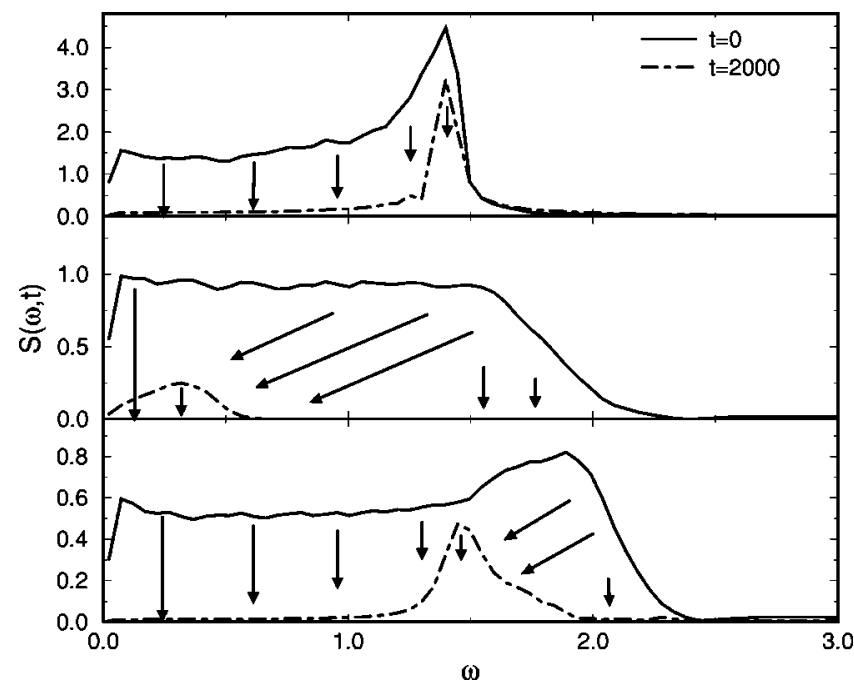

FIG. 4. Schematic representation of the spectral relaxation channels. The spectra of Fig. 3 for times $t=0$ and $t=2000$ are shown again here, and the arrows depict the pathways of different spectral components. Downward arrows indicate absorption by the cold reservoir, while angled arrows denote degradation from one spectral region to another. The relative lengths of the arrows represent the associated rates.
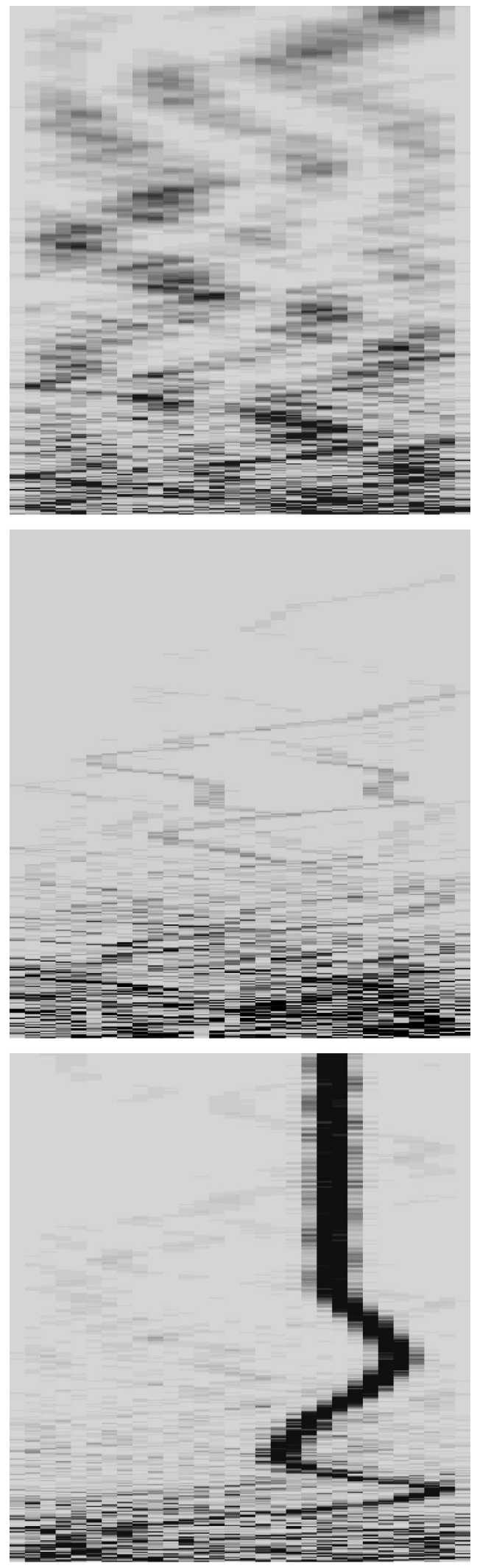

FIG. 5. Energy landscapes of 30-site arrays initially thermalized at $T=0.5$. Time advances along the $y$ axis until $t=1000$. A gray scale is used to represent the local energy, with darker shading corresponding to more energetic regions. First panel: harmonic chain, $k=0.5$ and $k^{\prime}=0$. Second panel: purely anharmonic chain, $k=0$ and $k^{\prime}=0.5$. Third panel: mixed chain, $k=k^{\prime}=0.5$. 
the first panel of Fig. 3 we show the spectrum of the harmonic chain at different times during the relaxation process, starting with the initial thermalized spectrum [in this figure $S(\omega, 0) \equiv S(\omega)$ of Fig. 1]. The evolution confirms that low frequencies decay more rapidly in the harmonic chain-the spectrum is absorbed by the cold reservoir from the bottom up, and by time $t=2000$ only the longer-lived band-edge modes remain in the system. The spectral decrease occurs "vertically," that is, each spectral component decays directly into the reservoir; this is shown schematically in Fig. 4, where the downward arrows represent absorption by the reservoir and their relative length schematizes the absorption rate. The first panel of Fig. 5 shows the time evolution of the local spatial energy landscape using a gray scale to contrast higher-energy (darker) from lower-energy (lighter) regions.

The purely hard array relaxes more rapidly than the harmonic and the energy decays essentially exponentially, indicating a single predominant decay channel. Note that being a purely quartic chain, there are no phonons in this system. We also observe in the second panel of Fig. 3 that higherfrequency excitations relax first. At time $t=2000$ only lowfrequency excitations remain in the chain. This behavior is exactly opposite to that of the harmonic chain. We find that the dominant relaxation mechanism is for the high-frequency portions of the spectrum to degrade into lower-frequency excitations, as schematically indicated by the sloped arrows in Fig. 4. Such a degradation is possible here since individual frequencies are not associated with normal modes in the anharmonic system. In turn, these lower-frequency excitations decay into the reservoir. Specifically, the high-frequency components of the spectrum are mainly due to mobile localized modes that degrade into lower-energy excitations as they move and collide with one another, and this degradation occurs with a relatively short-time constant that is shorter for localized modes that have a higher velocity. The lowerfrequency excitations are in turn absorbed into the cold reservoir but continue to be replenished through the degradation process. We conjecture that the absorption of the lowestfrequency components defines the observed exponential decay constant of the total chain energy. However, and importantly, among the low-frequency excitations are some that persist for a very long time, certainly beyond the times of our simulations. These, which are the only remaining spectral components at time $t=2000$, are "labeled" by short downward arrows in the relaxation schematic and include rather stable breather and/or soliton modes that move very slowly and are localized away from the boundaries. At the same time, some portion of the high-frequency spectrum is also directly absorbed by the cold reservoir (indicated by the short downward arrow in the high-frequency region of Fig. 4). For example, when a highly mobile localized excitation reaches a boundary it may be absorbed directly by the reservoir (or it may be reflected and return into the chain with some energy loss). The role of high-frequency mobile modes and of low-frequency slowly moving or stationary modes in this picture will be tested in more detail in the next section, where we explicitly inject a high-frequency localized mode into the array and observe the relaxation dynamics. We do note here that our picture is consistent with known facts about localized states. In particular, it is known that higherfrequency and/or higher amplitude localized modes can move at higher velocities $[18,21,25]$. It is also known that while in motion such modes lose energy through collisions with other excitations. Also, Fig. 2 shows a faster decay at higher temperatures, which is consistent with our observations elsewhere that the speed of an injected pulse (and therefore, we conjecture, the speed of a moving localized mode) in these arrays increases with temperature [22]. The energy landscape associated with these descriptions is shown in the second panel of Fig. 5.

Almost all FPU analyses in the literature involve arrays that include both harmonic and anharmonic contributions, but the distinct role of each has not been clarified. Our relaxation results show an interesting sequence of relaxation behaviors. At early times the mixed array relaxes more rapidly than the harmonic, because there are low-frequency excitations close to harmonic phonons (but note that phonons are no longer exact normal modes) and high-frequency excitations in the system. Energy relaxation and decay thus involves both of the mechanisms discussed above. Again, because initially the high-frequency modes move more rapidly at higher temperature, the early time decay is faster at higher temperatures. That both low- and high-frequency modes relax rapidly is clearly seen in the third panel of Fig. 3, which quickly loses both low-(as in the first panel) and high-(as in the second panel) frequency portions of the spectrum. In the energy decay curve there is then a crossing after which the mixed chain relaxes much more slowly than the harmonic and the purely anharmonic. This occurs when the lowfrequency modes (phonons) have essentially all decayed, and only certain high-frequency spectral components remain, as clearly seen in the spectrum. We conjecture that these persistent modes are localized quasistationary breathers that decay extremely slowly, and again pursue this notion in the next section. The associated decay schematic is illustrated in Fig. 4. Note that with increasing temperatures the stationary breathers are more energetic, leading to a slower decay of the total chain energy at long times. Indeed, we find that the very slow decay at long times is a stretched exponential, as shown in Fig. 6. Here we plot

$$
\beta(t)=\frac{d}{d \ln t} \ln \left[-\ln \left(\frac{E(t)}{E(0)}\right)\right] .
$$

If $E(t) / E(0)$ is of stretched exponential form $\exp \left[-(t / \tau)^{\sigma}\right]$, then $\beta(t)=\sigma$. Figure 6 shows the stretched exponential behavior for various arrays and clearly points to a $T$-dependent but $N$ - and $\gamma$-independent exponent $\sigma$. The decrease of $\sigma$ with increasing initial temperature is explained by the greater stability of more energetic breathers. The $N$ and $\gamma$ independence is explained by the fact that the rate-limiting step in the slow relaxation is the leakage of the breather. The lowenergy output of this leakage is quickly absorbed by the cold reservoir [40].

These results immediately raise a question that in hindsight pervades a number of results throughout the literature: if both the purely quartic chains and also the mixed chains support high-frequency localized solutions, why do these 


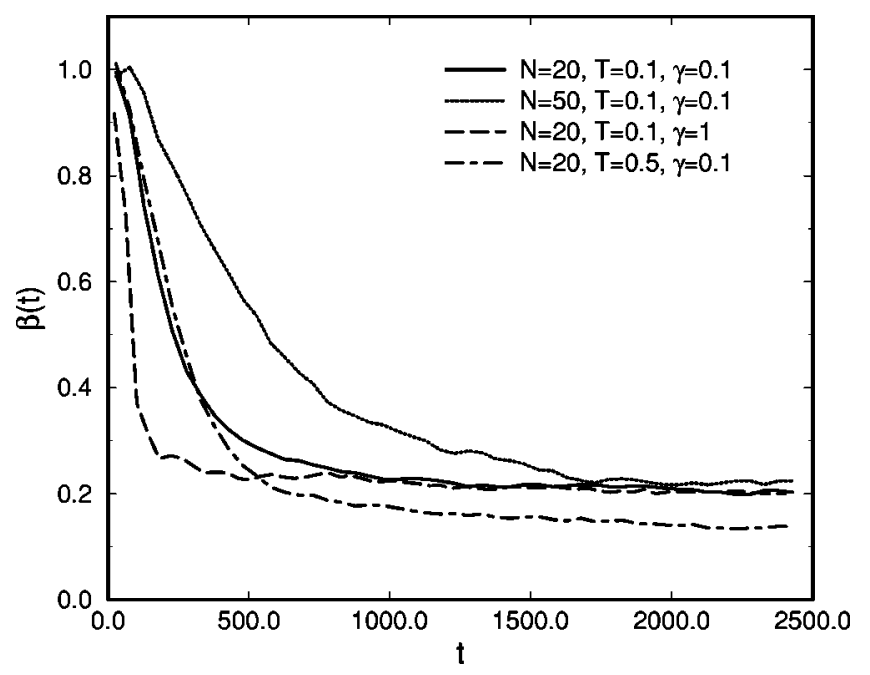

FIG. 6. Plot of $\beta(t)$ as a function of time for various mixed arrays. A flat line below $\beta(t)=1$ indicates stretched exponential behavior.

modes relax so rapidly in the former but seem to persist for very long times in the latter? The answer lies in the crucial role of the quadratic terms of the potential and the consequent behavior of the low-frequency portion of the spectrum. When localized solutions (breathers) are sufficiently strongly perturbed, they respond by moving $[18,21,25]$ and hence are subject to the degradation process described earlier. In a mixed chain, the low-frequency excitations (phonons) that can easily perturb breathers decay quickly, and at later times only the essentially unperturbed high-frequency breathers remain. These almost-stationary solutions of the system hardly move. Since localized breather modes tend to lose their energy only while they move, these quasistationary breathers can persist for a very long time. In the purely anharmonic chain, on the other hand, there are no phonons and the lowfrequency excitations include slowly-moving quasistationary anharmonic modes that persist for a long time and that continue to perturb the high-frequency localized modes. These energetic localized modes thus continue to move and collide with the low-frequency modes, resulting in degradation into lower-energy excitations.

The third panel in Fig. 5 shows the spontaneous appearance, slowing motion, and eventual stoppage of a breather in the mixed chain. It is this breather, absent from the purely hard array, that mainly leads to the persistent high-frequency spectral contributions and to the extremely slow relaxation of the mixed chain energy at long times.

\section{RELAXATION OF THERMALIZED ARRAY WITH AN INJECTED LOCALIZED EXCITATION}

In the previous section we portrayed a relaxation dynamic for anharmonic FPU arrays that involves a very specific view of the roles of high-frequency localized modes, lowfrequency anharmonic modes, and phonons. In order to further test these ideas, in this section we start again with the thermalized chain, but now we inject a high-amplitude localized excitation at time $t=0$ in the center of the chain. Spe-

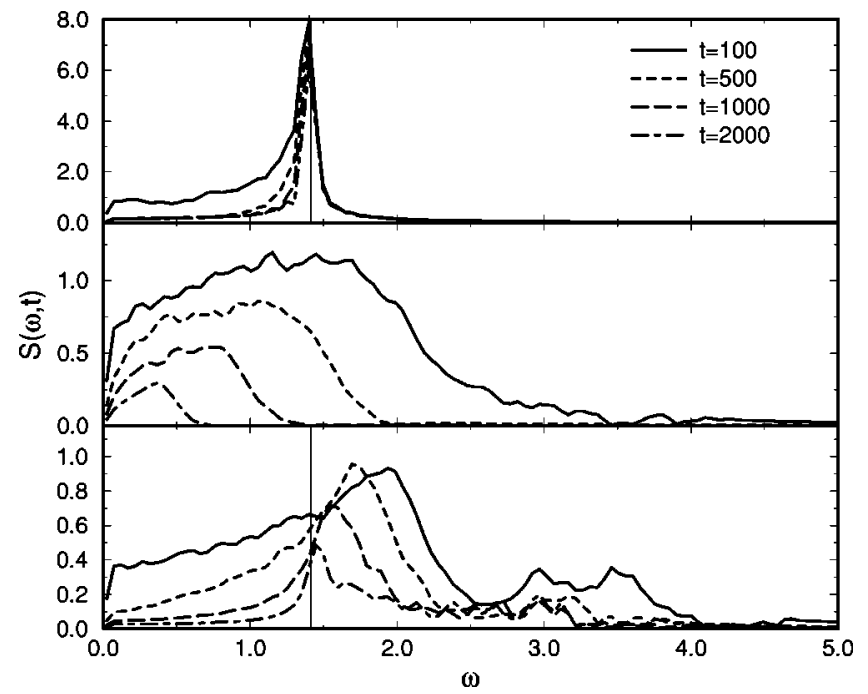

FIG. 7. Time evolution of spectra for various relaxing arrays initially at $T=0.1$ with a high-amplitude localized mode injected at $t=0$. First panel: harmonic chain with $k=0.5$. Second panel: purely anharmonic chain with $k^{\prime}=0.5$. Third panel: mixed chain with $k$ $=k^{\prime}=0.5$. The thin-vertical line again indicates the frequency $\omega$ $=\sqrt{4 k}=\sqrt{2}$. The $t=0$ spectra are not shown.

cifically, we inject an odd-parity excitation (amplitude $A$ at site $N / 2$ and $-A / 2$ at each immediately adjacent site). These displacements lead to an exact breather solution for the interaction potential $V\left(x_{i}-x_{i-1}\right)=\left(x_{i}-x_{i-1}\right)^{n}$ as $n \rightarrow \infty$ (as does the even-parity breather of amplitude $A$ at one site and $-A$ at an immediately adjacent site) and are quite close to exact for the quartic FPU potential $[42,43]$. The fate of the excitation as the entire system relaxes clarifies the roles of the different spectral components in the relaxation process. The excitation amplitude is sufficiently large $(A=2)$ to insure clear presence above the thermal background.

In the previous section we introduced the notion of localized modes as an important component in the thermal relaxation process of the FPU systems. We explicitly differentiated between mobile and stationary localized modes, and noted that energy loss occurs when a localized mode moves and collides with other localized modes. We also stated that this energy loss occurs through degradation into lowerfrequency modes. In order to focus on this mechanism without additional interference from the ends of the chain other than the normal low-frequency decay processes discussed earlier, in this section we use relatively long chains, $N$ $=300$. This is sufficiently long that we never see a highenergy localized mode reaching a boundary site before it has degraded or stopped moving.

The motion of the injected excitation during the relaxation process is followed in two ways. As before, we obtain the spectrum of the chains at various times (see Fig. 7). We also calculate the mean-squared displacement

$$
\left\langle x^{2}(t)\right\rangle \equiv\left\langle\left[i_{\max }(t)-\frac{N}{2}\right]^{2}\right\rangle
$$

as a measure of the position of the excitation (its dispersion in the anharmonic chains is very small [22]). Here $N / 2$ is the 


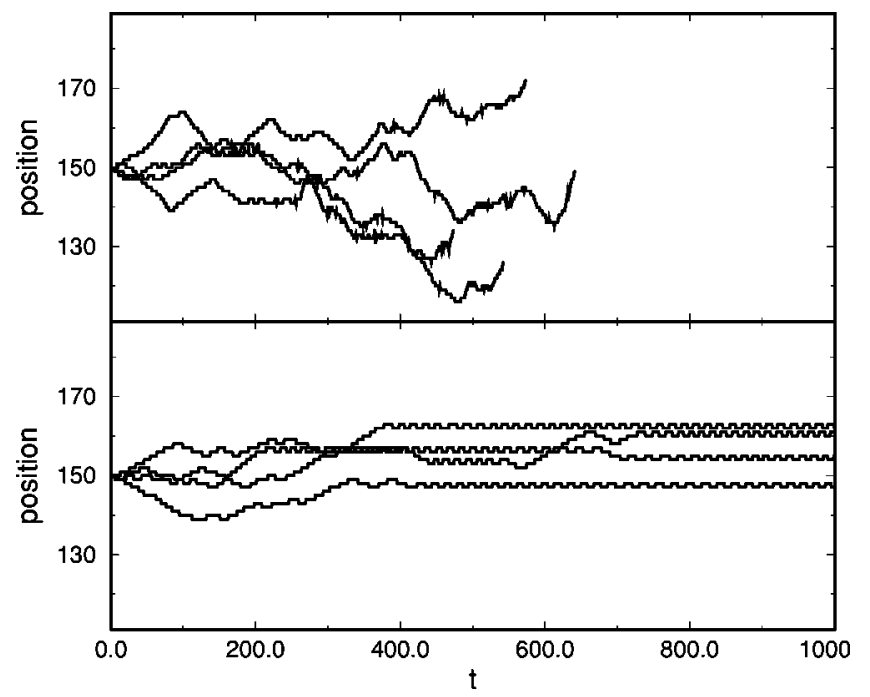

FIG. 8. Typical injected breather trajectories. Upper panel: purely anharmonic array. Lower panel: mixed array.

initial point of highest energy in the chain and $i_{\text {max }}(t)$ is the point of maximum energy at time $t$.

In a harmonic chain the progression is as expected for a harmonic system. The initial localized excitation spreads quickly over the entire array and thus loses its localized character. The associated Fourier decomposition into phonon modes dictates the relaxation behavior seen in Fig. 7, which is much like that seen in Fig. 3, except that the highfrequency (longer-lived) phonon modes are now more populated.

In the purely anharmonic chain, shown in the second panel of Fig. 7, the initial excitation introduces frequency components in a fairly broad spectral range (including frequencies well above the corresponding thermal range). Part of this spectral contribution is associated with an excitation that remains spatially localized (the rest appears because the injected excitation is not an exact mode of the thermalized chain; whereas the localized mode appears with each realization, the other spectral contributions vary somewhat in detail from one realization to another). Consistent with our description in Sec. III, the high-frequency components again relax quickly, indicating an energy degradation of the highfrequency excitations into lower-frequency modes. The detailed trajectory of the initial localized excitation is quite interesting, and a few particular realizations are shown in the upper panel of Fig. 8. After a short time the excitation begins to move in one direction or the other with equal probability (an initially even-parity excitation behaves very similarly but takes a longer time to begin to move because even-parity breathers are more stable in FPU chains). The motion continues for a period of random duration. Then the excitation stops moving for a random period, until it moves again in either direction for a random period of time. While stationary, we have observed that (whatever its initial configuration, even or odd) the excitation has even parity, but when it moves it alternates between even and odd parity. Furthermore, the excitation only loses energy while in motion. A detailed analysis reveals that the stationary excitation is per-

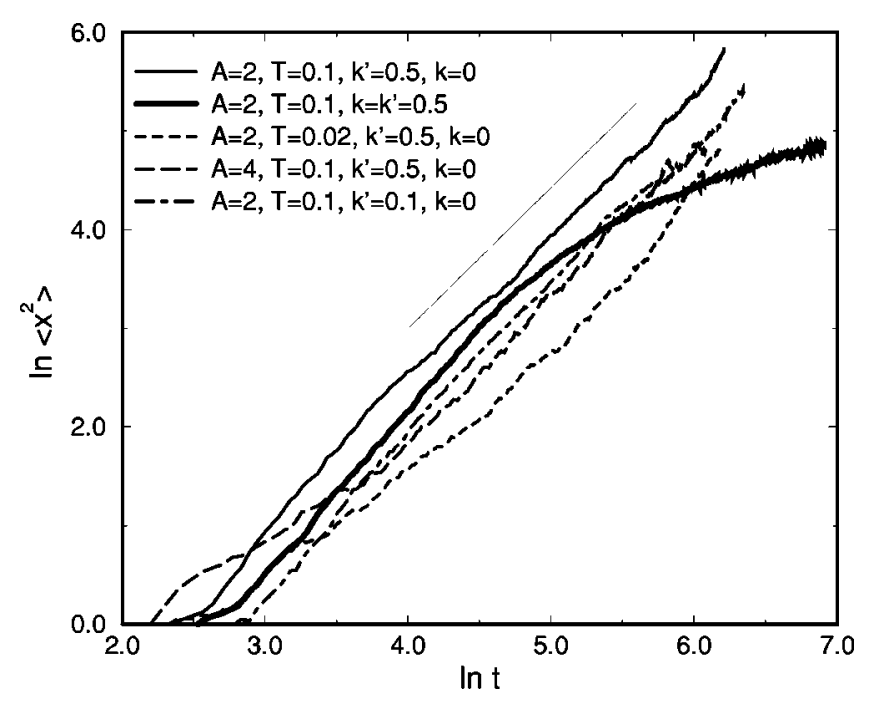

FIG. 9. Mean-squared displacement of a localized mode in various FPU arrays at various temperatures. The short straight line is a guide to the eye and has a slope of $3 / 2$.

turbed again and again by slow low-frequency localized excitations that collide with it and repeatedly set it in motion. These low-frequency modes are precisely those that, as described in the previous section, persist for a long time in the absence of a harmonic component. This sequence of events serves to confirm our thermalization analysis of Sec. III.

Mean-squared displacement results for a variety of parameter combinations are shown in Fig. 9. The mean-squared displacement is seen to follow the superdiffusive law $\left\langle x^{2}(t)\right\rangle \sim t^{\alpha}$ with $\alpha=3 / 2$ over the entire lifetime of the excitation. This particular exponent is recovered for the purely quartic chain under all conditions that we have tested, that is, independently of force constant, excitation amplitude, and temperature. Variations in parameters affect the breather velocity, which in turn modifies the coefficient of $t^{3 / 2}$, but not the power (a higher temperature, a stronger force constant, and a higher excitation amplitude all lead to higher velocities). Indeed, it does not even matter when in the course of the relaxation process the localized excitation is introduced: its mean-squared displacement follows the above behavior until the excitation is extinguished, again confirming that this behavior is mainly caused by the persistent low-frequency excitations.

A model that leads to the observed power law and contains the main features of the excitation collision picture was recently developed in a different context [44]. It describes a light particle that moves with a constant speed $\pm v$ among point scatterers arranged randomly on a line. The intervals between scattering points, $\xi_{n}$, are independent identically distributed random variables described by a probability density function $\mu(\xi)$. If the density decays as $\mu(\xi) \sim \xi^{-(1+\gamma)}$ with $1<\gamma<2$ when $\xi$ is large, then the mean-squared displacement of the light particle goes as $\left\langle x^{2}\right\rangle \sim t^{\alpha}=t^{3-\gamma}$. In particular, when $\gamma=3 / 2$ then $\alpha=3 / 2$ as in our results. This model, suitably modified, may describe our system. Our scatterers (low-energy breathers) do move, but all that is required to obtain the observed power law is that the times at which they collide with the injected excitation be distributed ac- 


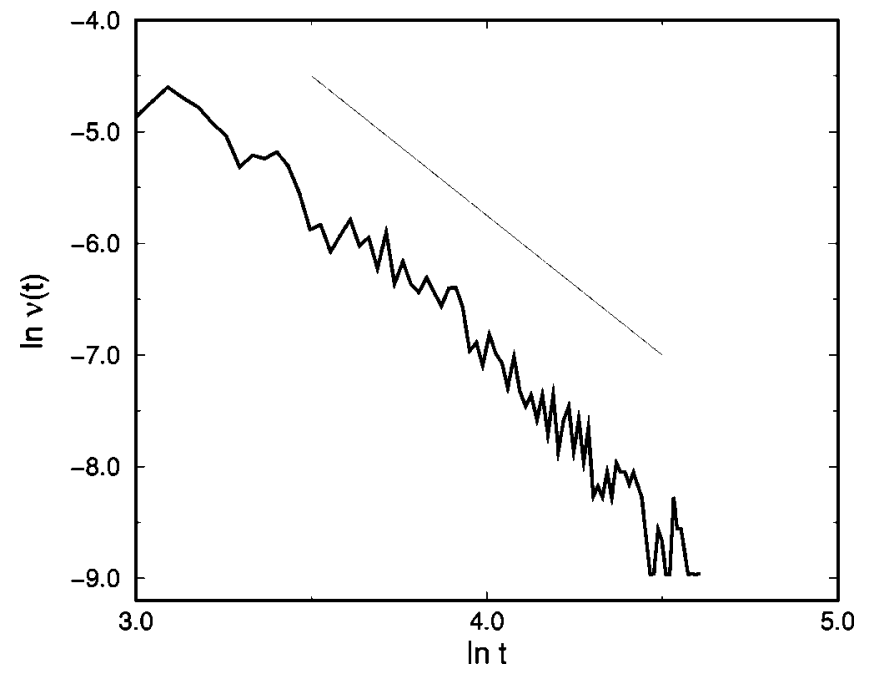

FIG. 10. Distribution of collision times of the high-frequency localized mode in the purely anharmonic FPU chain for times much greater than the typical oscillation period of the breather. The straight line has a slope of $-5 / 2$.

cording to $\nu(t) \sim t^{-5 / 2}$ (in the work of Barkai et al. [44], distance intervals and time intervals are interchangeable because the scatterers are stationary). The quiescent periods simply modify the coefficient of this and of the resulting mean-squared displacement distribution, but the power $\alpha$ is determined by the collision time distribution exponent. Numerical results for the distribution of times between breather collision events are shown in Fig. 10. The data is noisy (and could of course be made smoother with more realizations) but the confirming trend is clear. This is of course simply phenomenology, since we have no explicit dynamical model to obtain this distribution.

In the mixed chain, whose spectra are shown in the third panel of Fig. 7, the initial excitation again leads to the appearance and persistence of high-frequency spectral components. Typical trajectories of the highest-energy modes (which here, too, remain localized) are shown in Fig. 8. The difference between the purely anharmonic and the mixed typical trajectories are evident: whereas the excitations in the former continue to move until extinguished, the excitations in the mixed chain slow down and eventually stop altogether when all perturbing excitations have been swept out of the system. Once there are essentially no other excitations to collide with a stationary breather, it remains in the system in spite of the dissipative bath acting on its ends. This is completely consistent with the landscape shown in Fig. 7. The mean-squared displacement in Fig. 9 clearly reveals this behavior as well: the exponent begins at $3 / 2$ but eventually bends towards $\alpha=0$ when the breather stops moving.

\section{CONCLUSIONS}

In this paper we have studied energy relaxation in onedimensional nonlinear arrays with quartic interparticle interactions (Fermi-Pasta-Ulam or FPU arrays). In one scenario, we have thermalized the arrays to a temperature $T$ and then observed the relaxation of the arrays when the boundaries are connected to a zero-temperature reservoir through damping terms. In another scenario, we have introduced a high-energy localized excitation in the thermalized array and have observed the relaxation process and the fate of this excitation. This second scenario serves to confirm our description of the dynamics of thermal relaxation. Throughout we have applied free-end boundary conditions.

Our most salient results concern the role of harmonic contributions to the FPU potential as distinct from the thermalization of an array. In other words, we emphasize that one can equilibrate an array at a given temperature, whether or not its interactions include quadratic interactions, and we pose the following question: What exactly is the role of these interactions?

We have confirmed that thermal relaxation in a purely harmonic chain involves the sequential decay of independent phonon modes starting with those of lowest frequency and moving upward across the spectrum. We have also confirmed that the total energy of the array decays exponentially for short times and as an inverse power law at longer times, as calculated by Piazza et al [19]. When a localized excitation is introduced in a purely harmonic array it quickly spreads and loses any localized identity.

In a purely anharmonic chain there are no phonons, and the anharmonic excitations, in general, include localized modes. High-frequency spectral components include highly localized modes that may be stationary but are easily set in essentially ballistic motion by sufficiently strong scattering events. As a result, their net motion is superdiffusive. When not in motion these modes can retain their energy for a long time, but while in motion they lose energy through collisions with other excitations and eventually degrade into lowerenergy excitations. The lowest-energy excitations decay into the cold reservoir, while other low-energy excitations persist for a long time in the chain. The spectral relaxation proceeds mostly from the high-frequency end of the spectrum downward. At long times the energy residue that remains in the chain in the form of low-frequency localized excitations that move slowly is quite persistent but very small. To confirm this description we have observed the dynamics of an injected localized high-amplitude excitation. We observe that it is perturbed by the thermal excitations, which sets the breather in motion. This motion alternates with quiescent periods, but resumes when the excitation is again perturbed sufficiently strongly. Since during its lifetime there is always a slowly moving thermal background, the breather continues to resume motion until it disappears into the relaxing thermal background. The time dependence of the mean-squared displacement of the breather is remarkably universal over its entire lifetime, $\left\langle x^{2}\right\rangle \sim t^{3 / 2}$, independently of initial breather amplitude, temperature, and force constant.

In a mixed anharmonic array the relaxation process involves phononlike modes (with the lowest frequencies decaying first) and also high-frequency anharmonic modes (with the highest frequencies decaying first). The relaxation is at first rapid, but as the phonon decay "sweeps" the system clean of low-energy excitations, quasistationary highenergy breathers are no longer perturbed and remain essentially stationary; the subsequent relaxation process is 
exceedingly slow. When a high-energy localized excitation is injected in the mixed array, at first it is perturbed by thermal excitations that induce motion. However, as the thermal excitations are swept out of the system through the harmonic channel, the breather stops moving and survives for a very long time, thus confirming the relaxation picture. Associated with this description is a mean-squared displacement that at first goes as $\left\langle x^{2}\right\rangle \sim t^{3 / 2}$ but then becomes independent of time.

From a broader perspective, we have shown that vibrational energy localization and persistence is aided by the presence of an efficient mechanism to remove other background excitations that might perturb and/or destroy localization. In our specific model, localization is due to hard anharmonic interactions and the removal mechanism involves a harmonic phonon channel, but one can envision other local- ization and background sweeping processes that lead to a similar outcome. From a narrower perspective, there are a number of questions that remain to be explored, including relaxation in higher dimensions and the incorporation of more realistic potentials.

\section{ACKNOWLEDGMENTS}

This work was supported in part by the Engineering Research Program of the Office of Basic Energy Sciences at the U. S. Department of Energy under Grant No. DE-FG0386ER13606. Partial support was provided by a grant from the University of California Institute for Mexico and the United States (UC MEXUS) and the Consejo Nacional de Ciencia y Tecnología de México (CONACYT), and by IGPP under project Los Alamos/DOE 822AR.
[1] T. Pullerits and V. Sundstrom, Acc. Chem. Res. 29, 381 (1996); X. Hu and K. Schulten, Phys. Today 50, 28 (1997); Davydov's Soliton Revisited: Self-Trapping of Vibrational Energy in Protein, Vol. 243 of NATO ASI Series B: Physics, edited by P.L. Christiansen and A.C. Scott (Plenum, New York, 1990); K. Bolton, S. Nordholm, and H.W. Schranz, J. Phys. Chem. 99, 2477 (1995); L. Cruzeiro-Hansson and S. Takeno, Phys. Rev. E 56, 894 (1997).

[2] M.A. Collins, Adv. Chem. Phys. 53, 225 (1983); M.A. Collins and D.P. Craig, Chem. Phys. 75, 191 (1983); M.A. Collins, Phys. Rev. A 31, 1754 (1985); St. Pnevmatikos, N. Flytzanis, and M. Remoissenet, Phys. Rev. B 33, 2308 (1986); see the collection of papers in Phys. IV, Colloq. 5, 1155 (1995).

[3] M. Peyrard and J. Farago, Physica A 288, 199 (2000).

[4] G. Kopidakis and S. Aubry, Physica B 296, 237 (2001).

[5] T. Rössler and J.B. Page, Phys. Rev. B 62, 11460 (2000).

[6] S.F. Mingaleev, Y.S. Kivshar, and R.A. Sammut, Phys. Rev. E 62, 5777 (2000).

[7] S. Aubry and K. Kopidakis, e-print cond-mat/0102162.

[8] S. Flach and C.R. Willis, Phys. Rep. 295, 181 (1998).

[9] S. Aubry, Physica D 103, 201 (1997).

[10] X. Hu, A. Damjanovicz, T. Ritz, and K. Schulten, PNAS 95, 5935 (1998).

[11] H. Berg, Nature (London) 394, 324 (1998).

[12] W.O. Hancock and J. Howard, J. Cell Biol. 140, 1395 (1998).

[13] A. Xie, L. van der Meer, W. Hoff, and R.H. Austin, Phys. Rev. Lett. 84, 5435 (2000).

[14] B. Hu, B. Li, and H. Zhao, Phys. Rev. E 57, 2992 (1998).

[15] T. Prosen and D.K. Campbell, Phys. Rev. Lett. 84, 2857 (2000).

[16] K. Aoki and D. Kusnezov, Phys. Rev. Lett. 86, 4029 (2001).

[17] F. Zhang, D.J. Isbister, and D.J. Evans, Phys. Rev. E 61, 3541 (2000).

[18] R. Bourbonnais and R. Maynard, Int. J. Mod. Phys. C 1, 233 (1990).

[19] F. Piazza, S. Lepri, and R. Livi, nlin.CD/0105028.

[20] R. Reigada, A. Sarmiento, A.H. Romero, J.M. Sancho, and K.
Lindenberg, J. Chem. Phys. 112, 10615 (2000).

[21] T. Cretegny, T. Dauxois, S. Ruffo, and A. Torcini, Physica D 121, 109 (1998).

[22] A. Sarmiento, R. Reigada, A.H. Romero, and K. Lindenberg, Phys. Rev. E 60, 5317 (1999).

[23] R. Reigada, A. Sarmiento, and K. Lindenberg, Phys. Rev. E 63, 066113 (2001).

[24] V.M. Burlakov, S.A. Kiselev, and V.N. Pyrkov, Phys. Rev. B 42, 4921 (1990).

[25] Y.A. Kosevich and S. Lepri, Phys. Rev. B 61, 299 (2000).

[26] J.L. Marín, F. Falo, P.J. Martínez, and L.M. Floría, Phys. Rev. E 63, 066603 (2001).

[27] N.J. Zabusky and M.D. Kruskal, Phys. Rev. Lett. 15, 240 (1965).

[28] R. Dusi, G. Viliani, and M. Wagner, Philos. Mag. B 71, 597 (1995); Phys. Rev. B 54, 9809 (1996).

[29] Y.A. Kosevich, Phys. Rev. B 47, 3138 (1993).

[30] A.J. Sievers and S. Takeno, Phys. Rev. Lett. 61, 970 (1988).

[31] J.B. Page, Phys. Rev. B 41, 7835 (1990).

[32] K.W. Sandusky, J.B. Page, and K.E. Schmidt, Phys. Rev. B 46, 6161 (1992).

[33] T. Dauxois and M. Peyrard, Phys. Rev. Lett. 70, 3935 (1993).

[34] S. Aubry, Physica D 71, 196 (1994).

[35] R.S. MacKay and S. Aubry, Nonlinearity 7, 1623 (1994).

[36] D. Cai, A.R. Bishop, and N. Gronbech-Jensen, Phys. Rev. E 52, 5784 (1995).

[37] S. Takeno and M. Peyrard, Physica D 92, 140 (1996).

[38] R. Reigada, A. Sarmiento, M. Copelli, and K. Lindenberg (unpublished).

[39] G.P. Tsironis and S. Aubry, Phys. Rev. Lett. 77, 5225 (1996).

[40] A. Bikaki, N.K. Voulgarakis, S. Aubry, and G.P. Tsironis, Phys. Rev. E 59, 1234 (1999).

[41] J. DeLuca, A.J. Lichtenberg, and S. Ruffo, Phys. Rev. E 51, 2877 (1995).

[42] S. Flach, Phys. Rev. E 51, 1503 (1995).

[43] J.L. Marin and S. Aubry, Nonlinearity 9, 1501 (1996).

[44] E. Barkai, V. Fleurov, and J. Klafter, Phys. Rev. E 61, 1164 (2000). 